\title{
空间高光谱相机调焦机构精度分析与试验
}

\author{
王 凯 ${ }^{1,2}$ 徐明林 ${ }^{1}$ 解 鹏 $^{1}$ 徐 振 ${ }^{1}$ 陶淑苹 ${ }^{1}$ \\ (1. 中国科学院长春光学精密机械与物理研究所 长春 130033; \\ 2. 中国科学院大学 北京 100049)
}

\begin{abstract}
摘要: 针对空间高光谱相机的使用特性及要求, 设计一套调焦范围 $\pm 3 \mathrm{~mm}$ 的像面移动式调焦机构, 对偏离的焦面进行校正, 以满足高光谱相机成像质量要求。介绍调焦机构的工作原理, 对调焦机构的传动关系、定位精度进行理论分析; 建立调焦机 构的动力学模型, 进行有限元分析; 详细论述编码器码值与 CMOS 靶面位移关系测试方法, 得到真实可靠的调焦公式; 动力 学试验表明, 调焦机构一阶自然频率为 $182.7 \mathrm{~Hz}$, 可以有效地避免共振现象, 加速度响应合理, 具有较好的强度、刚度; 同 时在动力学试验前后, 对调焦机构分别进行闭环精度测试, 定位精度均优于 $\pm 6 \mu \mathrm{m}$, 试验结果表明调焦机构具有较好的稳定 性, 满足 $\pm 10 \mu \mathrm{m}$ 的设计指标要求, 验证调焦公式以及闭环控制程序设计的有效性。目前, 此套调焦机构已经成功在轨运行, 各项指标状态良好, 满足高光谱相机的成像质量要求。
\end{abstract}

关键词: 高光谱相机；调焦机构；精度分析；CMOS 靶面；动力学

中图分类号: TH12; V443

\section{Precision Analysis and Experiment of Focusing Mechanism of Space Hyperspectral Camera}

\author{
WANG Kai $^{1,2}$ XU Minglin ${ }^{1} \quad$ XIE Peng $^{1} \quad$ XU Zhen $^{1} \quad$ TAO Shuping $^{1}$ \\ (1. Changchun Institute of Optics, Fine Mechanics and Physics, \\ Chinese Academy of Sciences, Changchun 130033; \\ 2. University of Chinese Academy of Sciences, Beijing 100049)
}

\begin{abstract}
For meeting the features and requirements of space hyperspectral camera, the image surface movable focusing mechanism is designed, the motion displacement is in the range of $\pm 3 \mathrm{~mm}$, correcting deviated focal plane, and meeting the imaging quality requirements of hyperspectral camera. The working principle of the focusing mechanism is introduced, and the transmission curve and positioning accuracy are theoretically analyzed; Dynamic model of the focusing mechanism is established and the finite element analysis is performed. The relationship test method of code value and displacement of CMOS target plane is discussed in detail, and obtain the reliable focusing formula. The dynamic experiments show that the first-order natural frequency of the focusing mechanism is $182.7 \mathrm{~Hz}$, which can effectively avoid the resonance phenomenon, the acceleration response is reasonable, and has good strength and stiffness; Before and after the dynamic experiments, the closed-loop accuracy tests are performed on the focusing mechanism, and the positioning accuracy is better than $\pm 6 \mu \mathrm{m}$, therefore the focusing mechanism has good stability, which satisfies the $\pm 10 \mu \mathrm{m}$ design index and verifies the effectiveness of focusing formula and closed-loop control programs. At present, this set of focusing mechanism has been successfully on-orbit operation, and the indicators are in good condition, which satisfies the imaging quality requirements of hyperspectral camera.
\end{abstract}

Key words: hyperspectral camera; focusing mechanism; precision analysis; CMOS target plane; dynamics

\section{0 前言}

空间对地观测视野广阔、覆盖范围大，是获取

* 吉林省自然科学基金(20170101164JC)和吉林省优秀青年人才基金 (20180520193JH)资助项目。20180601 收到初稿, 20181204 收到修改稿
地面信息的重要手段，其基本方式是利用空间相机 对地成像。随着航天产业的持续发展, 遥感技术在 国民经济的各行各业得到了广泛应用。空间相机在 发射过程中会受到复杂力学环境(冲击、振动、过载 等)的影响, 以及相机在轨运行时, 由于大气压力、 温度、微重力等空间环境与相机焦平面在地面标定 
时相比发生变化, 使得相机焦平面产生离焦, 导致 成像质量下降。为了满足空间遥感相机成像质量的 要求, 相机在空间投入使用之前必须对偏离的焦面 进行校正 ${ }^{[1-4]}$ 。

空间相机常用的调焦方式主要有三种, 即光学 元件调焦、调焦镜调焦、像面移动式调焦。目前, 调焦机构的研究多针对于大口径、长焦面或高分辨 率等空间相机 ${ }^{[5-11]}$, 而对于多光谱或高光谱相机调 焦机构的研究较少 ${ }^{[12]}$ 。为满足空间高光谱相机的使 用特性及成像质量要求, 设计了一套像面移动式调 焦机构, 介绍了调焦机构的工作原理、传动理论分 析、定位精度理论分析等, 建立了调焦机构的动力 学模型, 对其进行了有限元分析及试验验证, 详细 论述了编码器码值与 CMOS 靶面位移关系测试方 法, 闭环精度测试及数据分析等。

\section{1 调焦方案及工作原理}

\section{1 调焦方案}

高光谱相机光路简图如图 1 所示, 空间相机在 轨运行时, 地面景物经光学系统后, 成像在 CMOS 靶面上。由于空间复杂环境等的影响, 相机焦平面 会产生不同程度的偏离, 所成的像将不能满足成像 质量要求。通过调焦机构推动带有 CMOS 感光器件 的焦面组件沿着光轴方向前后移动, 使 CMOS 靶面 与相机焦平面重合 ${ }^{[13]}$ 。

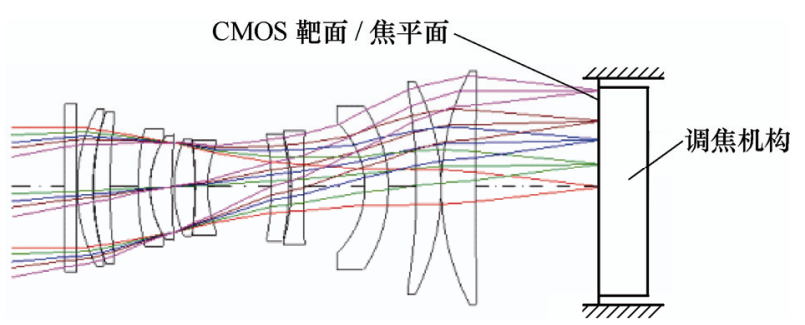

图 1 高光谱相机光路简图

相机光学系统具有一定的允许离焦量, 在焦面 深度范围内, 不会造成图像分辨率的下降。高光谱 相机的焦深

$$
\delta=4 F^{\# 2} \lambda=4 \times 5^{2} \times 0.45=45 \mu \mathrm{m}
$$

式中, $F^{\#}$ 为 $F$ 数; $\lambda$ 为光谱最低波段的中心波长。 调焦机构允许离焦量的最大值应为相机的半倍 焦深, 即 $\pm 22.5 \mu \mathrm{m}$, 考虑到空间复杂环境的影响, 将调焦精度设计值定为 $\pm 10 \mu \mathrm{m}$ 。经过分析计算, 同 时考虑结构设计时应使调焦量有一定的裕度 ${ }^{[14-15]}$, 将调焦量的设计值定为 $\pm 3 \mathrm{~mm}$ 。

\section{2 调焦工作原理}

如图 2 所示, 调焦机构主要由步进电动机、蜗
杆传动机构、螺旋传动机构、齿轮传动机构、编码 器(16 位)、焦面箱、精密直线导轨、支撑框架等组 成。步进电动机将动力传递到蜗杆, 带动蜗轮做旋 转运动, 蜗轮轴下部与丝杜形成螺旋副, 带动焦面 箱移动(焦面箱与丝杜通过螺钉连接)。为保证光学 靶面的直线性精度, 在焦面箱的两侧安装微型精密 级直线滚动导轨。同时, 蜗轮轴上部通过齿轮传动 机构与编码器连接, 实时反馈 CMOS 靶面的位置信 息, 以保证光学靶面的位置精度。

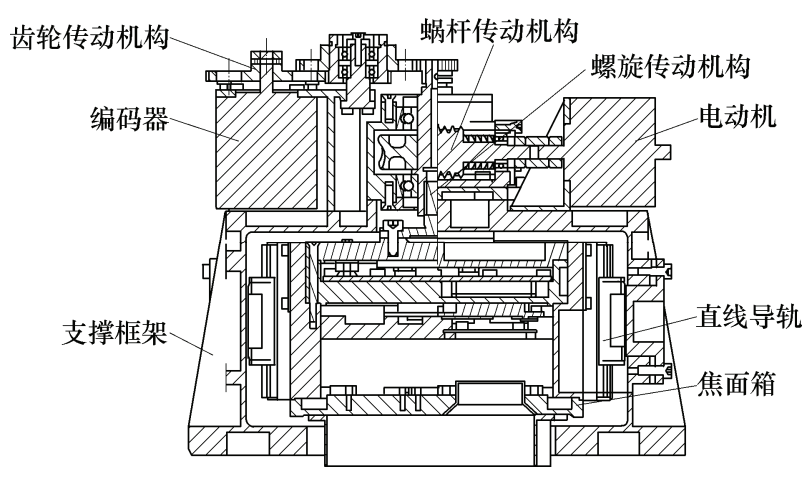

图 2 调焦机构

2 调焦机构理论分析

\section{1 传动理论分析}

如图 3 所示为调焦机构传动原理图。

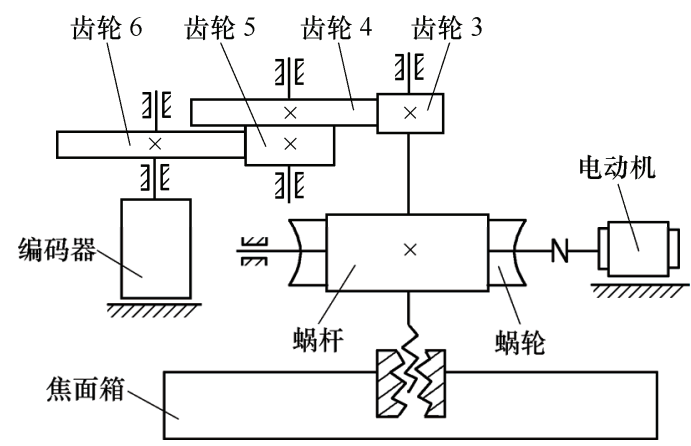

图 3 调焦机构传动原理图

调焦机构传动比

$$
i_{16}=\frac{z_{2} z_{4} z_{6}}{z_{1} z_{3} z_{5}}=\frac{42 \times 95 \times 95}{1 \times 30 \times 50}=\frac{2527}{10}
$$

式中, $z_{1}$ 为蜗杆头数; $z_{2}$ 为蜗轮齿数; $z_{3} 、 z_{4} 、 z_{5} 、 z_{6}$ 分别为齿轮 $3 、 4 、 5 、 6$ 的齿数。

丝杜螺纹的导程

$$
P_{h}=n P=1 \times 1=1 \mathrm{~mm}
$$

电动机旋转一圈(400 步), CMOS 靶面的位移量

$$
\Delta x=\frac{1}{i_{12}} p_{h}=\frac{1}{42} \times 1=\frac{1}{42} \mathrm{~mm}
$$

式中， $i_{12}$ 为蜗杆传动机构传动比。 
调焦机构灵敏度

$$
\Delta x_{\min }=\frac{\Delta x}{400} \approx 0.06 \mu \mathrm{m}
$$

电动机旋转一圈, 编码器的码值变化量

$$
\Delta y=\frac{1}{i_{16}} \times 2^{16}=\frac{655360}{2527} \approx 259
$$

斜率

$$
k=\frac{\Delta y}{\Delta x}=\frac{655360}{2527} \times 42 \approx 10892.41
$$

得到编码器码值 $y$ 与 $\operatorname{CMOS}$ 靶面位移 $x$ 的理论 关系曲线

$$
y=10892.41 x+a_{0}
$$

式中, $a_{0}$ 为 CMOS 靶面位于零位时编码器的码值。

\section{2 定位精度理论分析}

调焦机构采用闭环控制工作方式, 即编码器实 时反馈 CMOS 靶面的位置信息。由于 CMOS 靶面 与编码器之间的传动机构存在传动误差, 使编码器 未能真实反应 CMOS 靶面的实际位置信息 ${ }^{[16]}$, 由此 引入定位误差

$$
\sigma_{1}= \pm\left(E_{1}+E_{2}\right)= \pm 4.20 \mu \mathrm{m}
$$

式中, $E_{1} 、 E_{2}$ 分别为传动机构固有误差(齿轮实际齿 廓与理想齿廓的偏差)、跳动误差(齿轮实际旋转中 心与理想旋转中心的偏差)。

所选光电编码器 (16 位) 的分辨率为 $20^{\prime \prime}$, 因此 编码器运动一圈 ${ }^{[17]}$, 引入定位误差

$$
\sigma_{2}= \pm \frac{20^{\prime \prime}}{360^{\circ}} \times \frac{2^{16}}{k} \approx \pm 0.10 \mu \mathrm{m}
$$

程序设计时, 将调焦机构的控制阈值设置为 \pm 10 个码值, 因此引入定位误差

$$
\sigma_{3}= \pm \frac{\Delta y}{k}=\frac{10}{10892.42} \approx \pm 0.92 \mu \mathrm{m}
$$

将调焦机构传动误差、编码器误差、控制误差 合成, 得到 CMOS 靶面的定位精度

$$
\begin{gathered}
\sigma= \pm \sqrt{\sigma_{1}^{2}+\sigma_{2}^{2}+\sigma_{3}^{2}}= \\
\pm \sqrt{4.20^{2}+0.10^{2}+0.92^{2}}= \pm 4.30 \mu \mathrm{m}
\end{gathered}
$$

优于 $\pm 10 \mu \mathrm{m}$ 的设计指标要求。

\section{3 有限元动力学分析}

为了提前考察调焦机构的动力学特性, 建立其 有限元模型如图 4 所示, 共包含 36295 个单元。其 中, 支撑框架、焦面箱、直线导轨、丝杜、蜗轮轴、 蜗轮、蜗杆等采用 $3 \mathrm{D}$ 体单元; 齿轮、编码器、电
动机等采用质量点进行模拟, 并通过 MPC 将质量 点与支撑框架刚性连接。

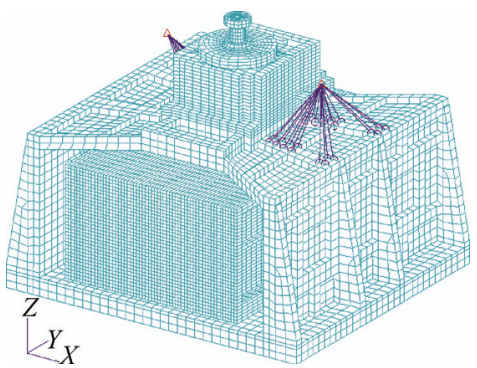

图 4 调焦机构有限元模型

\section{1 模态分析}

对调焦机构进行模态分析, 得到其前三阶自然频 率及振型如表 1 所示，一阶模态位移云图见图 5。阶基频为 $168.8 \mathrm{~Hz}$, 显著高于 $100 \mathrm{~Hz}$, 刚度分布合理。

表 1 调焦机构前三阶模态

\begin{tabular}{ccc}
\hline 阶数 & 频率 $/ \mathrm{Hz}$ & 振型 \\
\hline 一 & 168.80 & $Y$ 向摆动 \\
二 & 216.35 & $X$ 向摆动 \\
三 & 388.71 & $Z$ 向摆动 \\
\hline
\end{tabular}

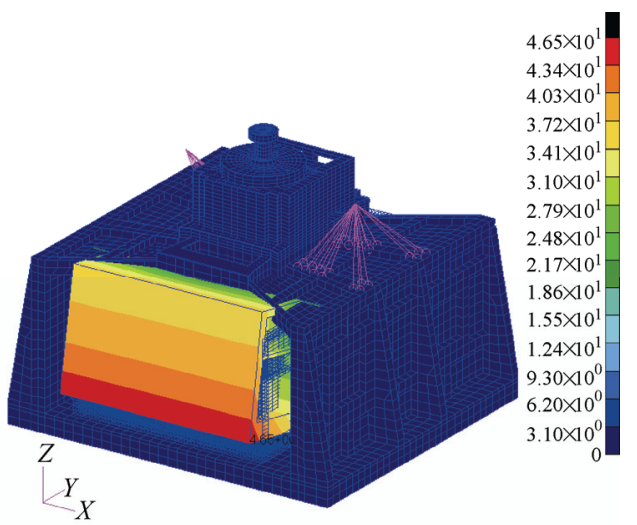

图 5 一阶模态位移云图

\section{2 频率响应分析}

分别沿 $X 、 Y 、 Z$ 三个方向对调焦机构进行频响 分析(频率域为 $10 \sim 100 \mathrm{~Hz}$, 加速度量级为 $1.5 \mathrm{~g} \sim$ $6 g$ )。其中, $Y$ 向加速度频率响应云图如图 6 所示, 在频率域内无明显的加速度响应, 响应范围在 $5.99 g \sim 6.96 g$, 最大放大倍率为 1.16 倍。焦面箱上 某点(Node 4 864, 与动力学试验传感器黏贴位置相 对应)的加速度频率响应曲线如图 7 所示。

\section{4 精度测试及分析}

调焦机构闭环控制方法主要有 2 种: (1) 使用同 一个调焦公式进行正、反向调焦; (2) 使用两个调焦 公式分别进行正、反向调焦。 


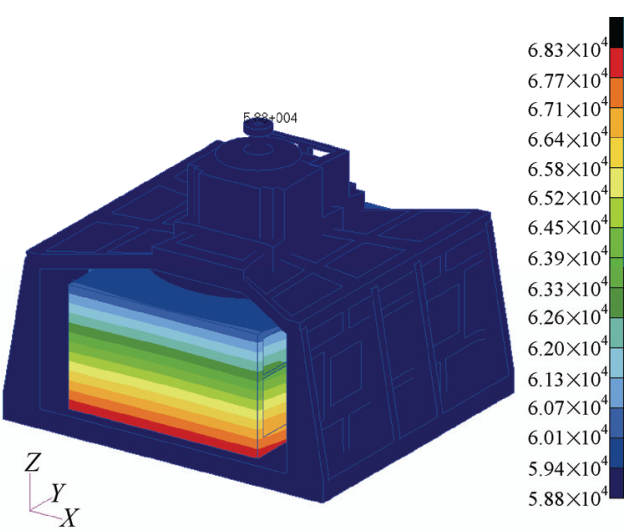

图 $6 Y$ 向加速度频率响应云图

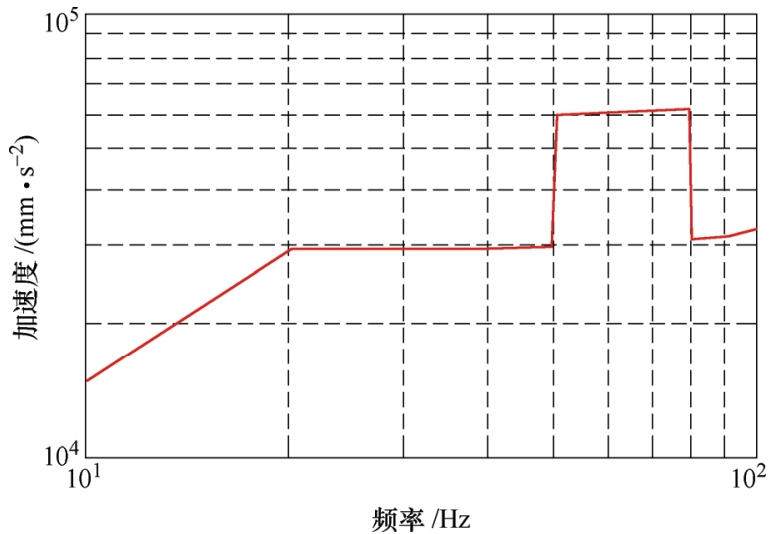

图 $7 Y$ 向加速度频率响应曲线

由于刚性机构固有误差等因素的影响，调焦机 构正、反向测试时, 位移与码值并不完全对应, 不 仅有系统误差(空回等)存在, 还有随机误差的影响。 同时, 数据拟合时也存在拟合误差, 故为了尽可能 地减少误差，采用第二种方法。

\section{1 码值与位移关系测试}

如图 8 所示, 首先对编码器码值与靶面位移关 系进行测试, 利用计算机发送指令控制 CMOS 靶面 沿光轴方向(垂直于 CMOS 靶面)移动, 同时读取编 码器码值、千分表数值变化。部分测试数据如表 2 所示。

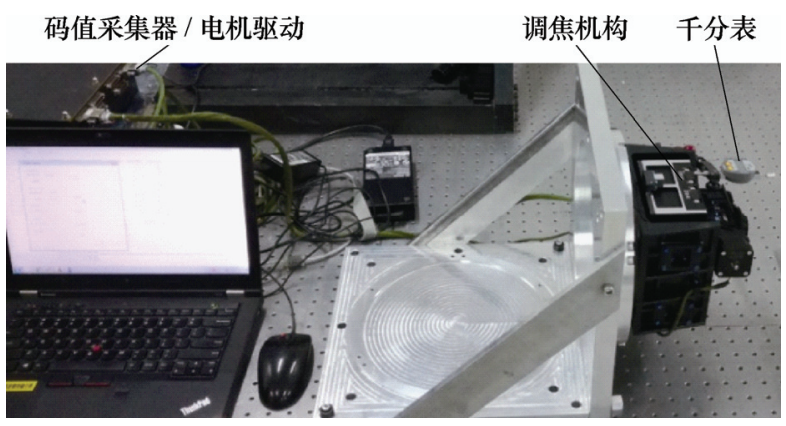

图 8 编码器码值与靶面位移关系测试
表 2 码值与位移测试数据

\begin{tabular}{ccc}
\hline 序号 & CMOS 靶面位移 $/ \mathrm{mm}$ & 编码器码值 \\
\hline 1 & -2.963 & 300 \\
2 & -2.367 & 6780 \\
3 & -1.777 & 13260 \\
4 & -1.190 & 19723 \\
5 & -0.588 & 26231 \\
6 & 0.000 & 32710 \\
7 & 0.585 & 39217 \\
8 & 1.176 & 45698 \\
9 & 1.768 & 52193 \\
10 & 2.363 & 58691 \\
11 & 2.954 & 65190 \\
\hline
\end{tabular}

以 CMOS 靶面位移为横坐标, 计算得到位移变 化量残差曲线、码值变化量残差曲线如图 9、10 所 示。由图可知位移变化量残差、码值变化量残差的 符号大体正负相同, 并且没有显著的变化规律, 故 判定测量数据无系统误差存在。

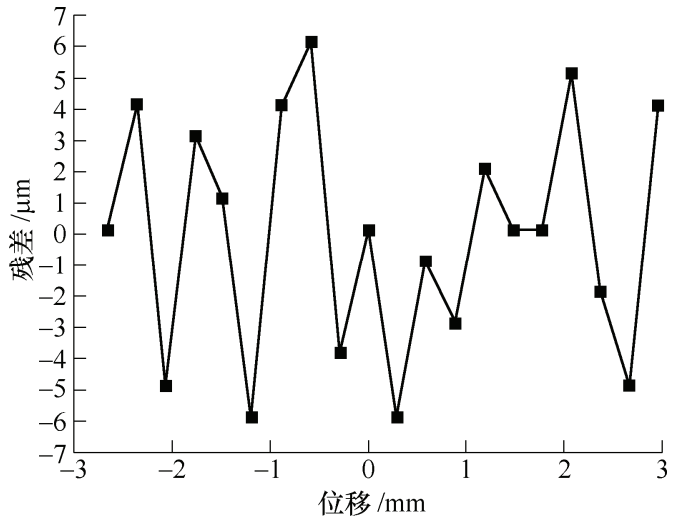

图 9 位移变化量残差曲线

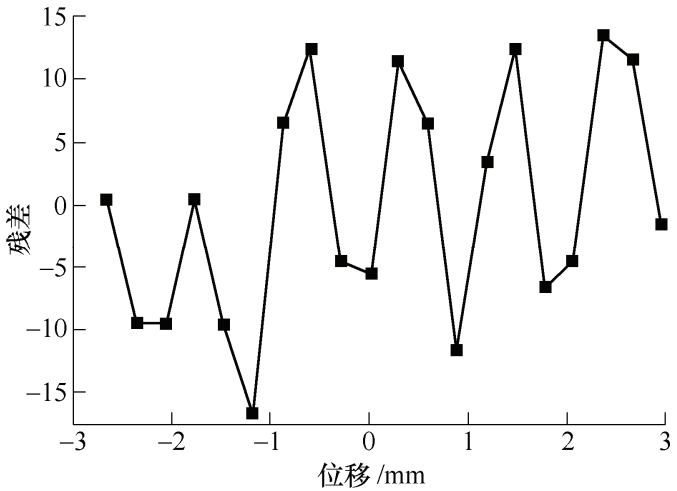

图 10 码值变化量残差曲线

位移变化量的标准差

$$
s_{\mathrm{d}}=\sqrt{\frac{\sum_{i=1}^{n} v_{i}^{2}}{n-1}}=3.8 \mu \mathrm{m}
$$

同理得码值变化量的标准差 $s_{c}=9.3$ 。

根据格罗布斯准则, 计算可知测量数据中不存 在粗大误差 


$$
\left|v_{i}\right| \geqslant G(\alpha, n) \cdot s
$$

式中, $v_{i}$ 为可疑数据的残差; 查表可得 $G(0.05,20)=$ 2.56 。

令 CMOS 靶面位移为变量 $x$, 编码器码值为变 量 $y$, 对测试数据线性拟合, 得到

$$
y=10975.10692 x+32770.32781
$$

如图 11 所示, 将式(15)与理论关系曲线式(8) 比较发现两条曲线基本吻合, 斜率 $k$ 的变化率

$$
\delta_{k}=\frac{\Delta k}{k}=\frac{82.69692}{10892.41}=0.759 \%
$$

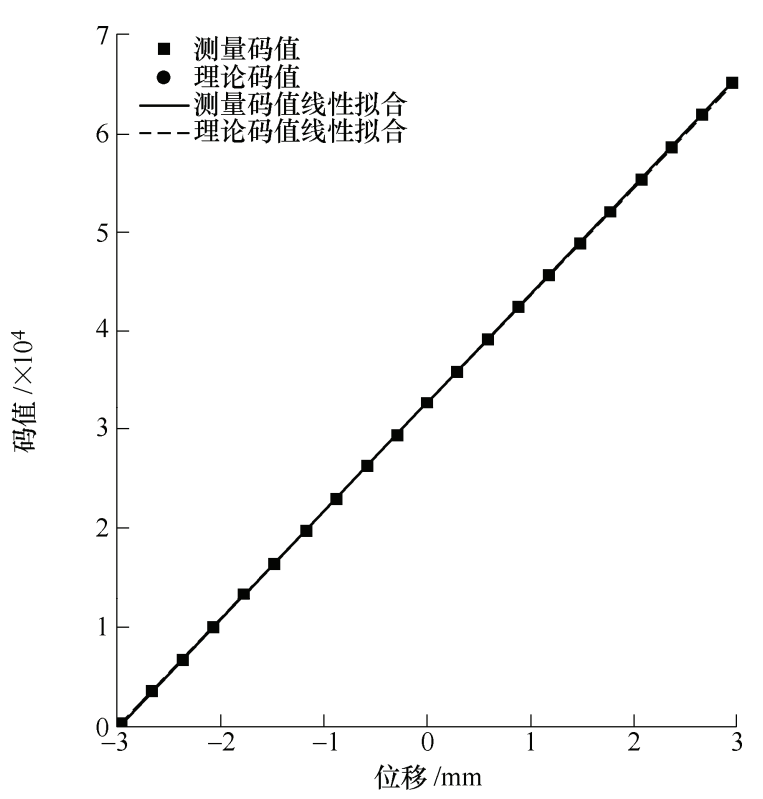

图 11 码值与位移线性关系曲线

同理得, 截距 $a$ 的变化率 $\delta_{\mathrm{a}}=0.184 \%$, 验证了 传动理论分析与线性拟合的正确性。

将拟合式(15)与表 2 测试数据相比较, 码值误 差在-86 49 范围内, 并以此为研究对象计算其标 准差 $s_{1}=38.78$ 。测量数据中系统误差、粗大误差均 可剔除, 但随机误差客观存在, 故测量数据可能呈 现非线性, 尝试对测试数据进行多项式拟合, 发现 变量 $x$ 的阶数在 $1 \sim 8$ 阶时, 拟合误差随阶数的增大 而明显变小; 大于 8 阶时, 拟合误差基本不变。八 次方拟合公式

$$
\begin{gathered}
y=-0.44974 x^{8}-0.61335 x^{7}+8.1485 x^{6}+ \\
10.42331 x^{5}-46.46408 x^{4}- \\
55.06837 x^{3}+91.80022 x^{2}+ \\
11061.05735 x+32726.59698
\end{gathered}
$$

拟合误差: 码值误差在 $-29 \sim 31$ 范围内, 标准差 $s_{8}=18.93$, 拟合精度明显高于式(15)线性拟合结果, 所以将式(17)作为闭环控制程序设计依据。其中, $x^{1}$ 的系数所占权重高达 $98.11 \%$, 即八次方拟合曲线
上每一点的曲率皆趋近于无穷大。线性拟合曲线、 八次方拟合曲线如图 12 所示。

$$
w_{1}=\frac{\left|b_{1}\right|}{\sum_{i=1}^{8}\left|b_{i}\right|}=\frac{11061.05735}{11274.0249}=98.11 \%
$$

式中, $w_{1}$ 为 $x^{1}$ 的系数所占权重; $b_{\mathrm{i}}$ 为 $x^{\mathrm{i}}$ 的系数。

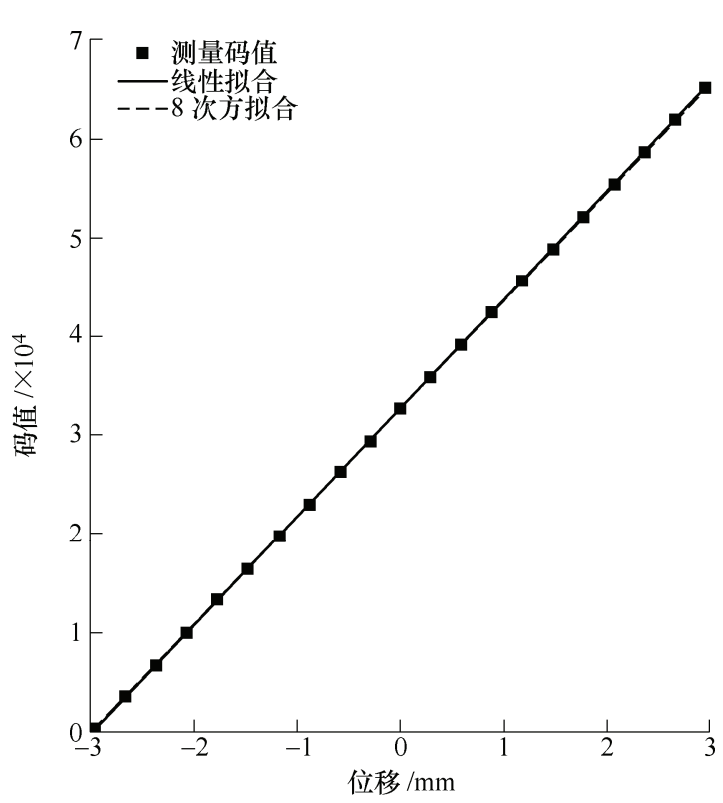

图 12 码值与位移线性、非线性(8 阶)拟合曲线

\section{2 精度测试及分析}

将式(17)作为调焦机构闭环控制程序设计 依据, 对其进行精度测试, 部分测试数据如表 3 所示。

表 3 闭环测试数据

\begin{tabular}{ccc}
\hline 序号 & CMOS 靶面位移 $/ \mathrm{mm}$ & 编码器码值 \\
\hline 1 & -2.964 & 289 \\
2 & -2.369 & 6780 \\
3 & -1.776 & 13277 \\
4 & -1.187 & 19728 \\
5 & -0.593 & 26205 \\
6 & 0.000 & 32727 \\
7 & 0.586 & 39223 \\
8 & 1.177 & 45734 \\
9 & 1.766 & 52145 \\
10 & 2.361 & 58678 \\
11 & 2.954 & 65192 \\
\hline
\end{tabular}

剔除闭环测试数据中的系统误差、粗大误差。 同时, 对闭环测试数据进行 8 次方拟合

$$
\begin{gathered}
y=-0.43457 x^{8}-0.58903 x^{7}+7.90975 x^{6}+ \\
10.02729 x^{5}-45.44537 x^{4}- \\
53.16736 x^{3}+90.95101 x^{2}+ \\
11058.68053 x+32726.328
\end{gathered}
$$


与式(17)相比较, 如图 13 所示, 吻合度极高, 其中截距 $a$ 的误差为 $0.0008 \%$; 占权重最高的 $x^{1}$ 的 系数 $b_{1}$ 的误差为 $0.02 \%$; 其余系数的误差在 $0.9 \%$ $4.0 \%$, 验证了调焦公式的正确性以及闭环控制程序 设计的有效性。

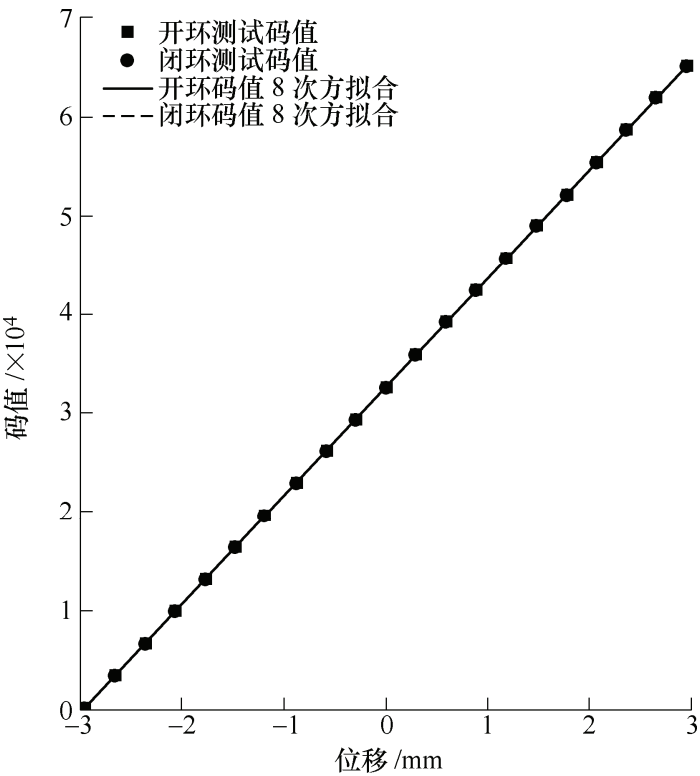

图 138 次方拟合曲线

调焦机构正向定位精度

$$
\sigma_{1}= \pm 3 s= \pm 5.2 \mu \mathrm{m}
$$

式中, $s$ 为闭环测试数据位移变化量的标准差。

同理可得调焦机构反向定位精度，此处不做赘 述。由于测量环境、测量仪器、人员操作以及调焦 机构本身的加工、装配误差等不确定性因素的影响, 由试验数据分析得到的定位误差大于理论定位误差 $\pm 4.30 \mu \mathrm{m}$, 并且满足了 $\pm 10 \mu \mathrm{m}$ 的调焦精度要求。

\section{5 动力学试验}

如图 14 所示, 分别沿 $X 、 Y 、 Z$ 三个方向对调 焦机构进行正弦扫频振动试验、正弦振动试验、随 机振动试验。

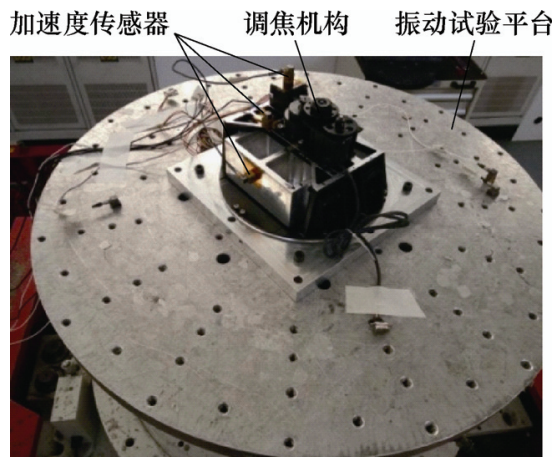

图 14 调焦机构振动试验

\section{1 扫频振动试验}

正弦扫频振动试验得到调焦机构自然频率如表 4 所示，响应曲线如图 15、16、17 所示。由于有限 元模型中, 单元划分时对复杂外形进行了部分简化, 同时部分结构采用质量点近似模拟，导致调焦机构 有限元分析得到的前三阶模态频率与试验得到的自 然频率存在一定的误差，分别为 $7.6 \% 、 4.8 \% 、 3.5 \%$,

表 4 调焦机构自然频率

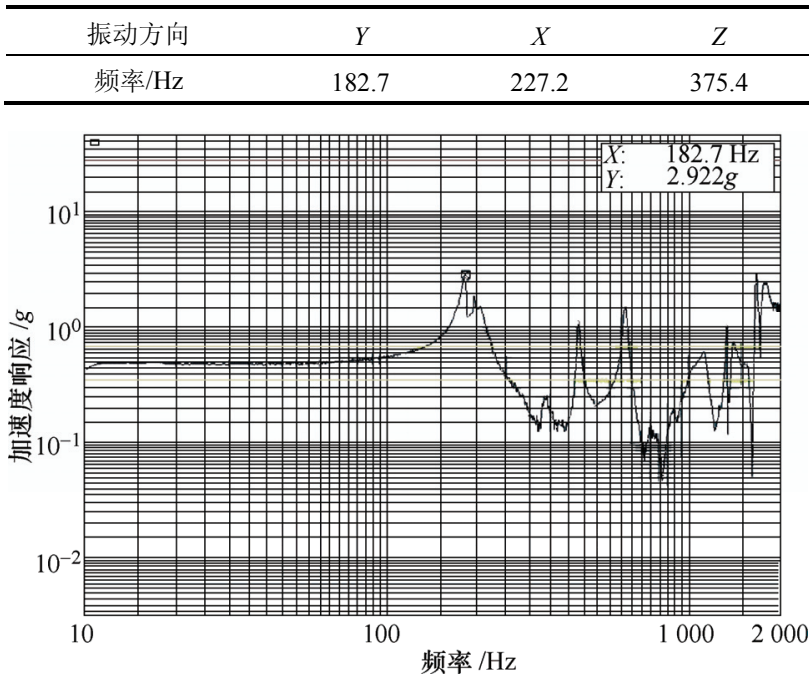

图 $15 Y$ 向扫频振动响应曲线

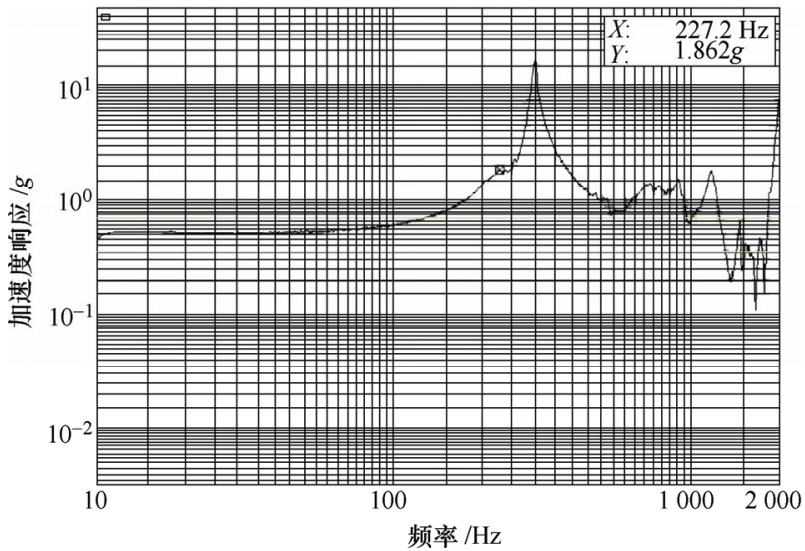

图 $16 X$ 向扫频振动响应曲线

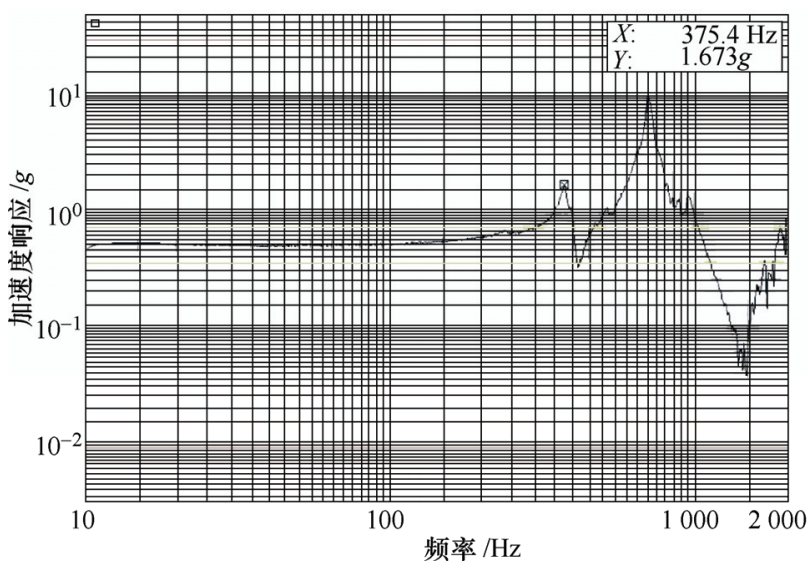

图 $17 Z$ 向扫频振动响应曲线 
均在 $8 \%$ 以内, 从工程应用的角度考虑是可以接受 的。试验得到的一阶自然频率为 $182.7 \mathrm{~Hz}$, 可以有 效地避免其在发射过程中与相机其他部分发生共振 现象。

\section{2 正弦振动试验}

对调焦机构进行正弦振动试验, 其中 $Y$ 向加速 度频率响应曲线如图 18 所示, $79.85 \mathrm{~Hz}$ 处加速度响 应最大为 $6.414 \mathrm{~g}$, 放大倍率为 1.07 倍, 与频率响应 分析结果基本吻合。同时，在 $10 \sim 100 \mathrm{~Hz}$ 频率域内， 调焦机构无明显的加速度响应, 进一步验证了调焦 机构 $Y$ 向基频大于 $100 \mathrm{~Hz}$ 。

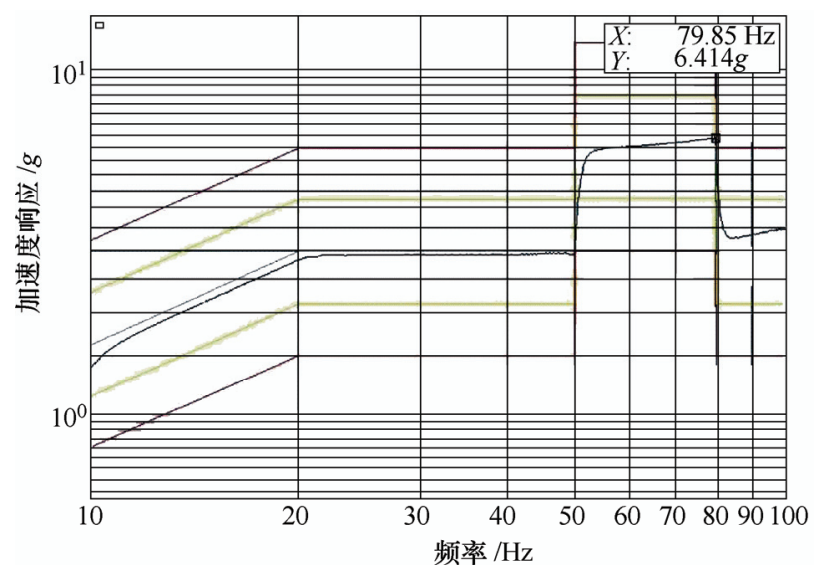

图 $18 Y$ 向加速度频率响应曲线

\section{3 随机振动试验}

对调焦机构进行随机振动试验(频率域为 20 $2000 \mathrm{~Hz}$, 加速度 RMS 为 7.05g), 其中 $Y$ 向功率谱 密度频率响应曲线如图 19 所示, $185 \mathrm{~Hz}$ 处响应最 大, 功率谱密度为 $8.595 \mathrm{~g}^{2} / \mathrm{Hz}$, 频率域内加速度总 方均根为 $23.41 \mathrm{~g}$, 放大倍率为 3.3 倍。

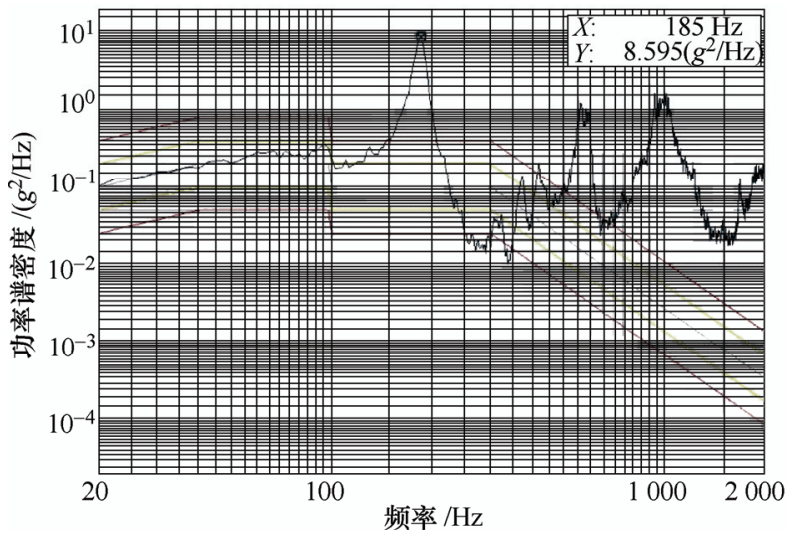

图 $19 Y$ 向功率谱密度频率响应曲线

动力学试验后, 对调焦机构进行外观检查, 未 发现变形、裂纹等现象, 说明调焦机构具有较好的 强度、刚度, 且试验结果与有限元动力学分析结果 基本吻合, 验证了动力学分析的正确性。最后, 重 新对调焦机构进行闭环精度测试, 调焦精度略有下
降, 且优于 $\pm 6 \mu \mathrm{m}$, 说明调焦机构具有较好的稳定 性, 满足了 $\pm 10 \mu \mathrm{m}$ 的调焦精度要求。

\section{6 结论}

针对空间高光谱相机调焦机构的使用特性及要 求, 设计了一套像面移动式精密调焦机构, 调焦范 围为 $\pm 3 \mathrm{~mm}$, 结论如下。

(1) 像面移动式调焦方案合理，技术可行。

（2）根据调焦机构传动误差、编码器误差、控 制误等合成, 得到 CMOS 靶面的理论定位精度 \pm 4.30 $\mu \mathrm{m}$, 优于 $\pm 10 \mu \mathrm{m}$ 设计指标要求。

（3）动力学试验表明, 调焦机构的一阶自然频 率为 $182.7 \mathrm{~Hz}$, 可以有效地避免共振现象, 与有限 元分析结果基本吻合，加速度响应合理，具有较好 的强度、刚度。

（4）在动力学试验前后, 分别对调焦机构进行 了闭环精度测试, 定位精度均优于 $\pm 6 \mu \mathrm{m}$, 试验结 果表明调焦机构具有较好的稳定性, 满足了 $\pm 10 \mu \mathrm{m}$ 的设计指标要求，同时验证了调焦公式以及闭环控 制程序设计的有效性。

（5）目前，此套调焦机构已经成功在轨运行, 各项指标状态良好, 满足了高光谱相机的成像质量 要求。

\section{参 考 文 献}

[1] 江浩. 超轻量化 $\mathrm{SiC}$ 反射镜及支撑技术研究[D]. 长春: 中国科学院长春光学精密机械与物理研究所, 2016.

JIANG Hao. Research on ultra-light $\mathrm{SiC}$ mirrors and support techniques[D]. Changchun: Changchun Institute of Optics, Fine Mechanics and Physics, Chinese Academy of Sciences, 2016.

[2] 丁亚林, 田海英, 王家骐. 空间遥感相机调焦机构设计 [J]. 光学精密工程, 2001，9(1): 35-38.

DING Yalin, TIAN Haiying, WANG Jiaqi. Design on the focusing mechanism of space remote-sensing camera[J] Optics and Precision Engineering, 2001, 9(1): 35-38.

[3] JIA Xuezhi, JIN Guang. Design and precision measurement of TDI CCD focal plane for space camera[J]. SPIE, 2012, 8419: 84190X-1-84190X-5.

[4] GAUNEKAR A S , WIDDOWSON Gary P , NARASIMALU Srikanth, et al. Design and development of a high precision lens focusing mechanism using flexure bearings[J]. Precision Engineering, 2005(29): 81-85.

[5] 贾学志, 张雷, 安源, 等. 空间光学遥感器精密调焦机 构设计与试验 $[\mathrm{J}]$. 机械工程学报, 2016, 52(13): 25-30. JIA Xuezhi，ZHANG Lei，AN Yuan, et al. Design and 
experiment research on precision focusing mechanism of space remote sensor[J]. Journal of Mechanical Engineering, 2016, 52(13): 25-30.

[6] 许志涛, 刘金国, 龙科慧, 等. 高分辨率空间相机调焦 机构精度分析 [J]. 光学学报, 2013，33(7)：0728001.

XU Zhitao, LIU Jinguo, LONG Kehui, et al. Accuracy analysis of focusing mechanism of high resolution space camera[J]. Acta Optica Sinica，2013，33(7): 0728001.

[7] 王书新, 李景林, 刘砧, 等. 大尺寸焦平面空间相机调 焦机构的精度分析 [J]. 光学精密工程, 2010, 18(10): 2239-2243.

WANG Shuxin, LI Jinglin, LIU Lei, et al. Accuracy analysis of focusing mechanism in space camera with long-focal-plane[J]. Optics and Precision Engineering, 2010, 18(10): 2239-2243.

[8] 伞兵, 李景林. 大口径反射光学系统调焦机构设 [J]. 红 外与激光工程, 2013, 42(增刊 2): 329-332.

SAN Bing, LI Jinglin. Focusing device design of the large aperture optics system[J]. Infrared and Laser Engineering, 2013, 42(Suppl.2): 329-332.

[9] 王忠善, 何欣, 崔永鹏, 等. 长焦面空间相机调焦机构 分析与验证 $[\mathrm{J}]$. 红外与激光工程, 2014, 43(4)： 1206-1209.

WANG Zhongshan, HE Xin, CUI Yongpeng, et al. Analysis and validation of large size focal-plane focusing device for space camera[J]. Infrared and Laser Engineering, 2014, 43(4): 1206-1209.

[10] 惠守文. 长焦距斜视实时航空相机自动调焦技术的研 究[D]. 长春: 中国科学院长春光学精密机械与物理研 究所, 2003.

HUI Shouwen. Study of automatic focusing technique for long focus oblique real-time aerial camera[D]. Changchun : Changchun Institute of Optics, Fine Mechanics and Physics, Chinese Academy of Sciences, 2003.

[11] ZHANG Xinjie, YAN Changxiang. Application of precision harmonic gear drive in focusing mechanism of space camera[J]. SPIE , 2010(7659) : 76590M-1-76590M-7.
[12] 吕世良, 刘金国, 贾平. 空间多光谱 CCD 相机调焦精 度分析 [J]. 红外与激光工程, 2013，42(2)：392-397.

LÜ Shiliang, LIU Jinguo, JIA Ping. Accuracy analysis of the focusing precision for multispectral CCD space camera[J]. Infrared and Laser Engineering, 2013, 42(2): 392-397.

[13] 刘否, 曹国华. 大视场长焦面光学遥感器双凸轮式焦面 调焦机构[J]. 光学精密工程, 2012, 20(9): 1939-1944. LIU Lei, CAO Guohua. Double cam focusing mechanism of space camera with wide field and long-focal-plane[J]. Optics and Precision Engineering, 2012, 20(9) : 1939-1944.

[14] 刘否. 空间三反相机调焦范围的确定 [J]. 光学精密工 程，2013，21(3): 631-636.

LIU Lei. Focusing range of space off-axial TMA optical camera[J]. Optics and Precision Engineering, 2013, 21(3): 631-636

[15] 王智, 张立平, 李朝辉, 等. 传输型立体测绘相机的调 焦机构设计 [J]. 光学精密工程, 2009, 17(5): 1051-1056. WANG Zhi, ZHANG Liping, LI Chaohui, et al. Design of focusing mechanism of space tridimensional mapping camera[J]. Optics and Precision Engineering, 2009, 17(5): 1051-1056.

[16] 张洪文. 空间相机调焦技术的研究[D]. 长春: 中国科学 院长春光学精密机械与物理研究所, 2003.

ZHNAG Hongwen. Research on the Auto-focusing technology for space camera[D]. Changchun: Changchun Institute of Optics, Fine Mechanics and Physics, Chinese Academy of Sciences, 2003.

[17] 王显军. 光电轴角编码器细分信号误差及精度分析 [J]. 光学精密工程, 2012, 20(2): 379-386.

WANG Xianjun. Errors and precision analysis of subdivision signals for photoelectric angle encoders[J]. Optics and Precision Engineering, 2012, 20(2): 379-386.

作者简介: 王凯, 男, 1992 年出生。主要研究方向为空间光学精密机械。 E-mail: wangkaiciomp@163.com 徐明林(通信作者), 男, 1988 年出生, 助理研究员。主要研究方向为空 间光学遥感器结构与机构设计。

E-mail: 18345172432@126.com 